\title{
Serum hepcidin measured by immunochemical and mass-spectrometric methods and their correlation with iron status indicators in healthy children aged 0.5-3 y
}

\author{
Lieke Uijterschout ${ }^{1}$, Dorine W. Swinkels², Magnus Domellöf ${ }^{3}$, Carina Lagerqvist ${ }^{3}$, Cisca Hudig ${ }^{4}$, Harrold Tjalsma ${ }^{2}$, Rimke Vos $^{5}$, \\ Johannes B. van Goudoever ${ }^{6,7}$ and Frank Brus ${ }^{1}$
}

BACKGROUND: The diagnostic use of hepcidin is limited by the absence of standardization and lack of age-specific reference ranges in children in particular. The aim of this study was to determine reference ranges of serum hepcidin in healthy children aged 0.5-3 y using mass spectometry (MS) and a commercial immunochemical (IC) assay, and to investigate its association with other indicators of iron status and inflammation.

METHODS: We included 400 healthy children aged 0.5-3 y. We constructed reference ranges for MS-hepcidin and IC-hepcidin concentrations using the median, P2.5, and P97.5 in a normative population of 219 children with no anemia, no infection and/or inflammation, and no iron deficiency.

RESULTS: Median concentrations (P2.5-P97.5) of MS-hepcidin and IC-hepcidin were $3.6 \mathrm{nmol} / \mathrm{I}(0.6-13.9 \mathrm{nmol} / \mathrm{I})$ and 7.9 $\mathrm{nmol} / \mathrm{l}(1.9-28.6 \mathrm{nmol} / \mathrm{l})$, respectively. We found a good correlation between both methods. However, MS-hepcidin was consistently lower than IC-hepcidin. Hepcidin correlated with ferritin and C-reactive protein.

CONCLUSION: We provide reference ranges for hepcidin for an MS and commercial IC method. Absolute values between assays differed significantly, but hepcidin concentrations obtained by MS and IC methods correlate with each other, and both correlate with ferritin and CRP.

$\mathrm{H}$ epcidin is a peptide hormone that is predominantly produced by hepatocytes. It circulates in the bloodstream and is excreted by the kidneys. Hepcidin plays a central role in regulating dietary iron absorption and body iron distribution. It induces the internalization and degradation of ferroportin, the major cellular iron exporter, resulting in increased intracellular iron stores, decreased dietary iron absorption and decreased circulating iron (1-3). Consequently, hepcidin has a bacteriostatic effect by reducing the plasma iron content, essential for microbial growth (2). The synthesis of hepcidin is decreased in situations that require increased concentrations of circulating iron such as iron deficiency (ID), hypoxia, ane$\mathrm{mia}$, and conditions characterized by increased erythropoietic activity $(3,4)$. A decrease in hepcidin results in the release of stored iron and an increase in dietary iron absorption. On the other hand, infection or inflammation causes an increase in hepcidin synthesis, which results in a decrease in iron available for erythropoiesis and contributes to anemia of chronic disease $(5,6)$. Although promising applications for hepcidin in diagnostic medicine have been described (2), they mostly apply to adults. Studies reporting hepcidin concentrations in children are scarce. Limited evidence suggests that hepcidin might contribute to the diagnosis of ID in preterm infants (7) and low birth weight infants (8) and could help to distinguish between iron deficiency anemia (IDA, low hepcidin) and anemia of chronic disease (high hepcidin) (9-12). Furthermore, hepcidin could be used in children to guide the effect of iron supplementation therapy and to screen for primary defects in hepcidin regulation such as iron refractory IDA (2).

However, the diagnostic use of hepcidin is limited by the absence of standardization in general, age-specific reference ranges of hepcidin and knowledge on the association between hepcidin and other iron status indicators in children in particular $(10,13,14)$. The aim of this study was to determine reference ranges of serum hepcidin in healthy children aged 0.5-3 y using Weak Cation eXchange Time of Flight mass-spectometry (MS) and an commercial immunochemical (IC) assay, and to investigate its association with other indicators of iron status and inflammation.

\section{RESULTS}

The study included 400 children (248 children from the Juliana Children's Hospital, 152 children from the Sophia Children's Hospital). Six children with underlying causes for anemia were excluded (Figure 1). Characteristics of the study population are described in Table 1. Median age was 16.5 mo. The study included significantly more boys than girls due to the types

\footnotetext{
'Department of Pediatrics, Juliana Children's Hospital/ HAGA Hospital, The Hague, The Netherlands; ${ }^{2}$ Department of Laboratory Medicine, Radboud University Medical Center, Nijmegen, The Netherlands; ${ }^{3}$ Department of Clinical Sciences, Pediatrics, Umeå University, Umeå, Sweden; ${ }^{4}$ Department of Clinical Chemistry, LabWest, HAGA Hospital, The Hague, The Netherlands; ${ }^{5}$ HAGA Academy, HAGA Hospital, The Hague, The Netherlands; ${ }^{6}$ Department of Pediatrics, VU University Medical Center, Amsterdam, the Netherlands; ${ }^{7}$ Department of Pediatrics, Emma Children's Hospital/Academic Medical Center, Amsterdam, The Netherlands. Correspondence: Lieke Uijterschout (l.uijterschout@hagaziekenhuis.nl or l.uijterschout@gmail.com)

Received 13 January 2014; accepted 30 April 2014; advance online publication 27 August 2014. doi:10.1038/pr.2014.109
} 


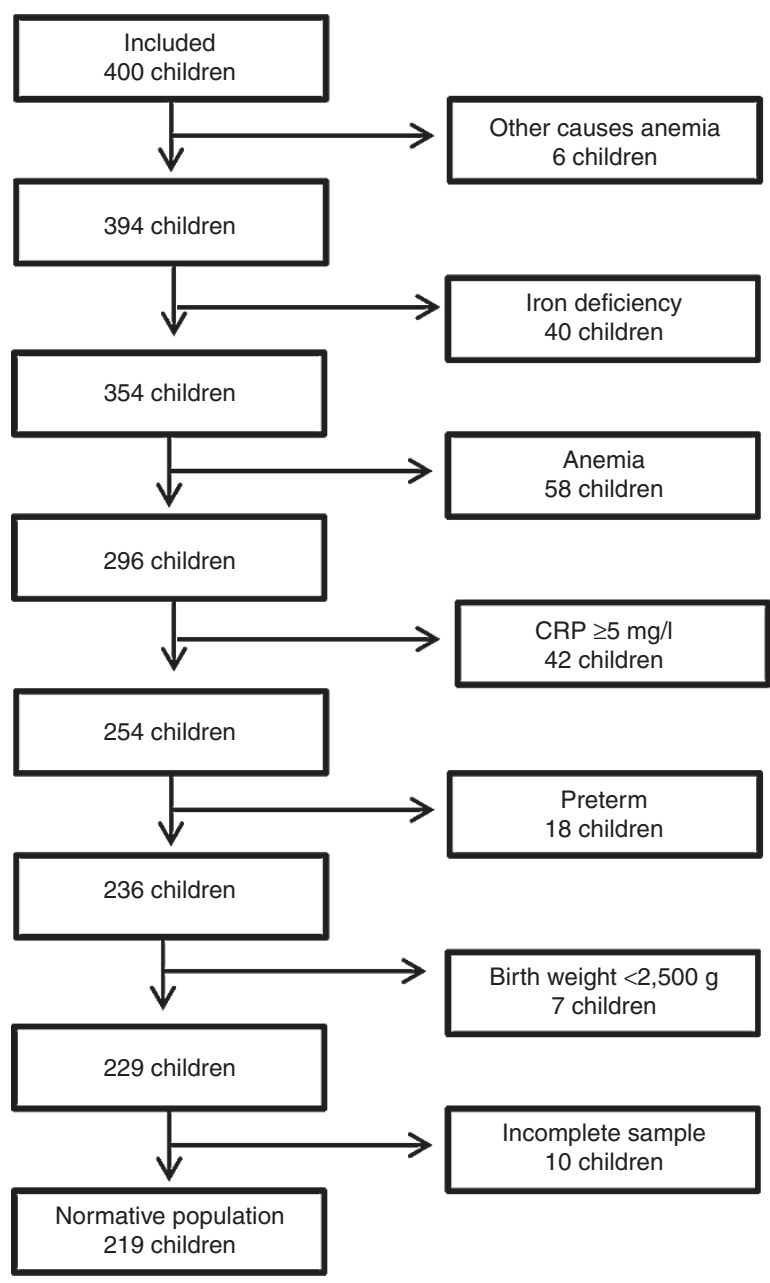

Figure 1. Flow chart of study population. CRP, C-reactive protein.

of surgical interventions that are carried out most often in healthy children at this age such as correction of hypospadia or orchidopexy (Table 1). Mean concentrations of all iron status indicators were similar in boys and girls (data not shown). Blood sampling time distribution over the age groups was dissimilar; older children underwent blood sampling more often later during the day. A total of 219 children were included in the normative population, to determine reference values (Figure 1).

\section{MS-Hepcidin and IC-Hepcidin Concentrations in the Normative Population}

Median concentrations (P2.5-P97.5) of MS-hepcidin and IC-hepcidin for the total study population were $3.6 \mathrm{nmol} / \mathrm{l}$ $(0.6-13.9 \mathrm{nmol} / \mathrm{l})$ and $7.9 \mathrm{nmol} / \mathrm{l}(1.9-28.6 \mathrm{nmol} / \mathrm{l})$, respectively. MS-hepcidin concentrations were consistently lower compared with IC-hepcidin concentrations. MS-hepcidin concentrations were lower than the detection limit of 0.5 $\mathrm{nmol} / \mathrm{l}$ in four children (1.8\%). We observed no differences in hepcidin between boys and girls (data not shown). Median hepcidin concentrations in children who underwent blood sampling in the morning were lower compared with hepcidin concentrations in children who underwent blood
Table 1. Characteristics of the study population

\begin{tabular}{|c|c|c|c|c|}
\hline Variable & $N$ & $\%$ & Median & P2.5-P97.5 \\
\hline Age (months) & 394 & & 16.5 & $6.0-34.0$ \\
\hline Gender (male) & 281 & 71.3 & NA & NA \\
\hline Birth weight (grams) & 394 & & 3,340 & $2,271-4,569$ \\
\hline Birth weight $<2,500 \mathrm{~g}$ & 22 & 5.6 & NA & NA \\
\hline Gestational age (weeks) & 394 & & 39.7 & $36.0-42.0$ \\
\hline Preterm (born <37 wk GA) & 23 & 5.8 & NA & NA \\
\hline Body mass index (SDS) & 394 & & -0.02 & -2.65 to 2.06 \\
\hline Socio-economical status & 394 & & -0.16 & -1.85 to 3.18 \\
\hline $\begin{array}{l}\text { Educational level of the } \\
\text { mother (high) }\end{array}$ & 157 & 39.8 & NA & NA \\
\hline Ethnicity (Western) & 273 & 69.3 & NA & NA \\
\hline \multicolumn{5}{|l|}{ Time of blood sampling } \\
\hline Before $12 \mathrm{pm}$ & 217 & 55.1 & NA & NA \\
\hline Between $12 \mathrm{pm}$ and $5 \mathrm{pm}$ & 177 & 44.9 & NA & NA \\
\hline \multicolumn{5}{|l|}{ Type of surgical intervention } \\
\hline Urological surgery & 127 & 32.2 & NA & NA \\
\hline General surgery & 80 & 20.3 & NA & NA \\
\hline ENT surgery & 75 & 19.0 & NA & NA \\
\hline Plastic surgery & 47 & 11.9 & NA & NA \\
\hline $\begin{array}{l}\text { Other surgical intervention } \\
\text { or diagnostic procedure }\end{array}$ & 65 & 16.5 & NA & NA \\
\hline Ferritin $(\mu \mathrm{g} / \mathrm{l})$ & 394 & 100 & 19.8 & $6.1-60.9$ \\
\hline $\operatorname{sTfR}(\mu \mathrm{g} / \mathrm{ml})$ & 393 & 99.7 & 5.5 & $3.0-15.7$ \\
\hline $\mathrm{Hb}(\mathrm{g} / \mathrm{l})$ & 394 & 100 & 111 & $90-129$ \\
\hline$(\mathrm{mmol} / \mathrm{l})$ & 394 & 100 & 6.9 & $5.6-8.0$ \\
\hline $\mathrm{MCV}(\mathrm{fL})$ & 394 & 100 & 77 & $69-84$ \\
\hline Ret-Hb (fmol) & 320 & 81.2 & 1.8 & $1.4-2.1$ \\
\hline $\begin{array}{l}\text { Iron deficiency } \\
\text { (ferritin }<10 \mu \mathrm{g} / \mathrm{l})\end{array}$ & 40 & 10.2 & NA & NA \\
\hline \multicolumn{5}{|l|}{ Infection/inflammation } \\
\hline CRP 0-4 mg/l & 343 & 87.1 & NA & NA \\
\hline CRP 5-30 mg/l & 51 & 12.9 & NA & NA \\
\hline
\end{tabular}

CRP, C-reactive protein; ENT, ear, nose, and throat; GA, gestational age; $\mathrm{Hb}$, hemoglobin; $\mathrm{MCV}$, mean corpuscular volume; Ret-Hb, mean $\mathrm{Hb}$ content in reticulocytes; SDS,

standard deviation score; STRR, soluble transferrin receptor.

sampling in the afternoon (Table 2, MS-hepcidin $P$ value $<0.001$, IC-hepcidin $P$ value 0.007$)$. Reference ranges for MS-hepcidin and IC-hepcidin concentrations in the normative subpopulation, stratified for age are shown in Table 2 , and stratified for both age and time of blood sampling in Supplementary Table S1 online.

\section{Correlations of MS-Hepcidin and IC-Hepcidin}

We found a significant correlation between MS-hepcidin and IC-hepcidin (Supplementary Figure S1 online). MS-hepcidin concentrations were almost 3 times lower than those obtained with the IC-asay ( $P$ value $<0.001$, intraclass correlation coefficient 0.568). The Blant-Altman plot showed that differences in observed concentrations were larger for the higher 
Table 2. Reference ranges stratified for age for MS-hepcidin and IC-hepcidin of children in the normative population ( $N=219)$

\begin{tabular}{|c|c|c|c|c|}
\hline & \multirow[b]{2}{*}{$N$} & \multirow[b]{2}{*}{ Median } & \multicolumn{2}{|c|}{$95 \% \mathrm{Cl}$} \\
\hline & & & P2.5 & P97.5 \\
\hline \multicolumn{5}{|c|}{ MS-hepcidin (nmol/l) } \\
\hline $0.5-1$ y & 64 & 3.7 & 0.3 & 18.2 \\
\hline $1-3 y$ & 155 & 3.6 & 0.7 & 14.6 \\
\hline \multicolumn{5}{|c|}{ IC-hepcidin (nmol/I) } \\
\hline $0.5-1$ y & 64 & 10.1 & 2.4 & 32.3 \\
\hline $1-3 y$ & 155 & 7.3 & 1.9 & 27.1 \\
\hline
\end{tabular}

hepcidin concentrations (Figure 2). Both MS-hepcidin and IC-hepcidin were significantly correlated with ferritin and CRP (Supplementary Tables S2 and S3 online). These correlations persisted after adjustment for age and time of blood sampling (Table 3 and Supplementary Table S4 online). We found no association between MS-hepcidin or IC-hepcidin and sTfR, Ret-Hb, MCV, and Hb (Table 3 and Supplementary Table S4 online). In a final multivariate model with hepcidin as dependent variable and ferritin, CRP, age and time of blood sampling as independent variables, we found that ferritin and CRP were independent correlates of hepcidin, which explained 39.9\% and $38.8 \%$ of the variance in MS-hepcidin and IC-hepcidin, respectively.

\section{DISCUSSION}

We present for the first time age-specific reference ranges for healthy children aged $0.5-3$ y, for serum hepcidin concentrations measured by mass spectrometry and an immunochemical assay. Hepcidin levels obtained by the two methods strongly correlated, but IC-hepcidin concentrations were consistently higher compared with MS-hepcidin concentrations, particularly in the high concentration range. Hepcidin levels obtained by either method strongly correlated with ferritin and CRP. These latter parameters together explained almost $40 \%$ of the total variance in hepcidin

Median MS-hepcidin concentrations in our normative subpopulation were $3.4-4.3 \mathrm{nmol} / 1$ and $2.6-4.0 \mathrm{nmol} / 1$ in children aged $0.5-1$ and $1-3 \mathrm{y}$, measured in the morning and afternoon, respectively. Studies that used the same mass-spectometry method to measure hepcidin, reported lower concentrations in 0.5 -y-old children in rural Kenya (mean $2.2 \mathrm{nmol} / \mathrm{l})(10)$ and in 11 -y-old children in Italy (mean $1.9 \mathrm{nmol} / \mathrm{l}$ ) (12). The lower concentrations in Kenyan children are likely attributed to a higher prevalence of ID. Furthermore, differences in age and ethnicity may have contributed to the observed differences in hepcidin concentrations between populations. Although prevalence of ID in the Italian study was lower than in our population, all blood samples were drawn earlier in the morning $(8 \mathrm{am})$, which might have resulted in lower hepcidin concentrations considering the diurnal rhythm of hepcidin $(15,16)$.

Median IC-hepcidin concentrations in our normative study population were $9.9-11.0 \mathrm{nmol} / \mathrm{l}$ in children aged

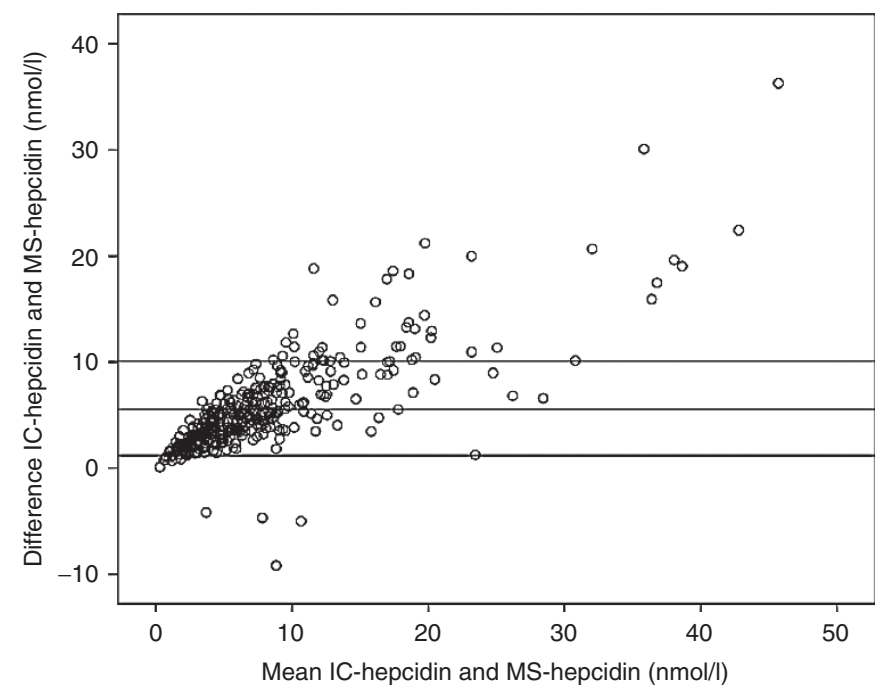

Figure 2. Blant-Altman plot of MS- and IC-hepcidin.

Table 3. Results of linear regression analyses for MS-hepcidin concentrations (nmol/l) adjusted for age and time of blood sampling ${ }^{\mathrm{a}}$

\begin{tabular}{lcccccc} 
& & \multicolumn{2}{c}{$95 \% \mathrm{Cl}$} & Adjusted & \\
\cline { 3 - 6 } Variable & \multicolumn{1}{c}{$\beta$} & $\mathrm{P} 2.5$ & $\mathrm{P} 97.5$ & \multicolumn{1}{c}{$\beta$} & $\mathrm{R}^{2}, \%$ & $P$ value \\
\hline Ferritin $(\mu \mathrm{g} / \mathrm{l})$ & 0.166 & 0.135 & 0.197 & 0.474 & 26.8 & $<0.001$ \\
$\mathrm{sTfR}(\mu \mathrm{g} / \mathrm{ml})$ & -0.063 & -0.226 & 0.100 & -0.038 & 4.8 & 0.45 \\
Ret- $\mathrm{Hb}(\mathrm{fmol})$ & -0.292 & -5.483 & -0.899 & -0.079 & 8.0 & 0.16 \\
$\mathrm{MCV}(\mathrm{fL})$ & 0.042 & -0.098 & 0.182 & 0.030 & 4.8 & 0.56 \\
$\mathrm{Hb}(\mathrm{g} / \mathrm{l})$ & -0.033 & -0.088 & 0.021 & -0.062 & 5.0 & 0.23 \\
$\mathrm{CRP}(\mathrm{mg} / \mathrm{l})$ & 0.581 & 0.477 & 0.685 & 0.484 & 28.0 & $<0.001$
\end{tabular}

$\mathrm{Cl}$, confidence interval; CRP, C-reactive protein; $\mathrm{Hb}$, hemoglobin; MCV, mean

corpuscular volume; MS, mass spectrometry; Ret- $\mathrm{Hb}$, mean $\mathrm{Hb}$ content in reticulocytes; sTfR, soluble transferrin receptor.

aTime of blood sampling was analyzed dichotomously as before or after $12 \mathrm{pm}$.

0.5-1 y, measured in the morning and afternoon respectively. Compared with IC-hepcidin concentrations (same manufacturer and cat-no, different batch-no) in unsupplemented low-birth-weight infant aged 0.5 y (17), hepcidin concentrations observed in our study were higher (geometric means $9.5 \mathrm{nmol} / 1$ in our study and $4.7 \mathrm{nmol} / 1$ in low-birth-weight infants), suggestive for lower prevalence of ID or a higher prevalence of infection in the former infants. However, the latter explanation is less likely since we used a slightly lower cut-off value for CRP to identify children with infection compared with the study in low-birth-weight $(5 \mathrm{mg} / \mathrm{l}$ and $8 \mathrm{mg} / \mathrm{l}$, respectively). Since hepcidin is sensitive to aggregation, easily sticks to laboratory plastic and the assays are not standardized, difference in hepcidin results between both studies might also be attributed to differences in pre-analytical sample handling and batches employed (2).

In accordance with other studies performed in children, ferritin explained almost $40 \%$ of the variance in hepcidin $(7,10,17)$. However, this percentage was less than half than in a large study in adults in which hepcidin was measured by an in house c-ELISA (18). On the other hand, contribution of CRP 
to the variance in hepcidin concentration in our study was slightly higher compared to results in adults $\left(R^{2} 25.0-28.0 \%\right.$ in our study and $20.0 \%$ in adults) (18). Even in children with a $\mathrm{CRP}<5 \mathrm{mg} / \mathrm{l}$, hepcidin was slightly but significantly associated with CRP after adjustment for age and time of blood sampling ( $R^{2} 7.0 \%$ for MS-hepcidin and $R^{2} 10.5 \%$ for IC-hepcidin, respectively). Similar results were observed in other studies performed in 0.5-y-old children (10). These results suggest that a higher contribution of CRP on the variance in hepcidin concentrations reflects the higher influence of infection and/ or inflammation on hepcidin concentrations in young children compared with adults.

We found a good correlation between the two methods, but levels of MS-hepcidin were almost three times lower than those of IC-hepcidin. These findings are in agreement with those found in recent round robin (send out of samples) of adult samples that also include the here used MS and EIA kit (cat nr. No S-1337), coded as MS-1 and IC-5, respectively (19). More specifically, in this round robin the WCX-TOF MS measured 2.89 times higher than the Bachem kit (Cat. No S-1337), whereas we measured larger differences between the two methods in samples with a higher hepcidin concentration. In general, hepcidin assay results are not traceable to reference materials and/or reference measurement procedures because neither reference materials nor a reference measurement procedure exists (19). Consequently, the differences in hepcidin concentrations between MS and IC assays in our study might be attributed to differences in the values that laboratories and companies assign to the internal and external standards used by the different methods, to impurities in these standards or to loss of the standard during storage, e.g., by aggregation $(20,21)$ or differential sticking of the synthetic hepcidins to the tubes (19). In this study, we attribute the differences between the assays in the fact that the respective companies assigned a lower value to the Bachem standard (used in the IC assay) than to the Peptide International standard (used in the MS method) (22), resulting in the higher the measurement results in the Bachem assay. This is supported by previous observations of lower values than expected for the pure standards of Bachem compared to those of Peptide International $(19,22)$. Consistent with the results from this round robin (19), our results illustrate the need for harmonization of different methods.

Hepcidin has been suggested as biomarker that might contribute to the diagnosis of ID (7-12), to guide the effect of iron supplementation therapy, and to screen for primary defects in hepcidin regulation (2). Reference values described in this study are instructive for investigating these potentials of hepcidin in future studies.

A limitation of our study is that hepcidin concentration in our population of healthy children living in the Southwestern region of the Netherlands might not be representative for children aged $0.5-3 \mathrm{y}$ in other populations. Although we carefully selected a normative population, both ID and infection/ inflammation are common conditions in children at this age. Suboptimal iron stores or minimal signs of infection/inflammation might have influenced hepcidin concentrations in our study. Furthermore it should be mentioned that we included more boys than girls in our study. However, we suggest that this did not influence our results since no gender effect was observed.

Taken together, to the best of our knowledge, this is the first study that extensively describes reference values of hepcidin in children aged $0.5-3$ y. The age-specific reference ranges presented in this study are instructive for the use of hepcidin as a diagnostic tool and therapeutic target in future studies. Our results on correlation of hepcidin concentrations with indicators of iron metabolism, erythropoiesis, and infection/inflammation contribute to our understanding of hepcidin regulation in young children. We showed that although hepcidin concentrations measured with WCX-TOF mass-spectometry and a commercial c-ELISA immunochemical differ significantly in absolute values, hepcidin concentrations obtained by MS and IC methods correlate with each other, and both correlate with ferritin and CRP. Future studies on harmonization of hepcidin assays are needed to enable comparison of hepcidin levels between studies as well as the definition of universal clinical decision limits.

\section{METHODS}

\section{Study Design}

To construct reference ranges for serum WCX-TOF MS hepcidin (MS-hepcidin) and IC derived hepcidin (IC-hepcidin) concentrations, we defined an iron-replete normative population by selecting healthy children born after $37 \mathrm{wk}$ gestational age (GA), a birth weight of with $\geq 2,500 \mathrm{~g}$, no anemia ( $\mathrm{Hb} \geq 100 \mathrm{~g} / \mathrm{l})$, no infection and/ or inflammation $(\mathrm{CRP}<5 \mathrm{mg} / \mathrm{l})$ and no ID (ferritin $\geq 10 \mu \mathrm{g} / \mathrm{l}$ ) (Figure 1) $(23,24)$. Reference ranges for MS-hepcidin and IC-hepcidin concentrations were defined as the P2.5 and P97.5 in children of the iron-replete normative population. All children were analyzed to investigate the correlations between hepcidin and other indicators of iron status and inflammation.

\section{Study Population}

We included 400 children aged $0.5-3$ y undergoing general anesthesia because of simple elective surgery or diagnostic procedure in two different hospitals in the Southwestern region of the Netherlands between August 2011 and May 2012 (25). This was originally a crosssectional study on prevalence and risk factors of ID. Blood samples of the aforementioned children have been used in the current study. During a pre-operative screening an extensive medical history and physical examination were performed by an anesthesiologist or an experienced pediatric resident, in order to include only healthy children. Children referred for adenotomy or tonsillectomy were not included because the prevalence of upper airway infections in these children is high. Exclusion criteria were known infection in the last $4 \mathrm{wk}$, use of iron supplementation within the last $6 \mathrm{wk}$, blood transfusion within the last $6 \mathrm{mo}$, preterm birth before $32 \mathrm{wk}$ gestational age (GA), known hemoglobinopathies, oncologic disorders, multiple congenital malformations and metabolic diseases as described previously (25). The study was approved by the Medical Ethics Committee of South-West Holland. All parents of the participating children gave written informed consent.

\section{Data Collection}

All children received a peripheral venous catheter to administer anesthetics. During insertion of the catheter, venous blood $(3 \mathrm{ml})$ was collected with an EDTA and a serum tube, and analyzed for ferritin, C-reactive protein (CRP), hemoglobin ( $\mathrm{Hb})$, mean corpuscular volume (MCV) and mean hemoglobin content in reticulocytes (Ret-Hb). We measured Ret-Hb since it has been reported that this is an early indicator of iron-restricted erythropoiesis (26). Samples were 
centrifuged and serum was aliquoted and frozen at $-80{ }^{\circ} \mathrm{C}$ until measurement of soluble transferrin receptor (sTfR) and hepcidin. Blood samples were drawn between 8 am and $8 \mathrm{pm}$ and time of blood sampling was recorded. Length was measured to the nearest millimeter by using a stadiometer or a length board in children who were not able to stand. Birth weight and gestational age were recorded and mothers of participating children were asked to give written informed consent for access to their own medical records to verify this information. Weight was measured to the nearest $0.1 \mathrm{~kg}$ by using a digital scale. Length, weight, and body mass index (BMI) were expressed as standard deviation scores (SDS) (27). Parents were asked to fill out a questionnaire concerning demographic data, parental education and information on the child's diet. Educational level of the parents was classified as "low" when they attended primary education, "intermediate" or "high" when at least one of them completed intermediate or higher vocational education/university, respectively. When both parents were born in a Western country (Europe, United States, and Oceania) children were classified as "Western." Socioeconomic status (SES) was determined by postal code. SES scores are available for each of the 3876 four-digit postal code areas in the Netherlands for the year 2010. SES scores are provided by The Netherlands Institute for Social Research (a Dutch governmental organization) and based on the following items (i) mean annual income per household, (ii) the percentage of households with a low income, and (iii) the percentage of households with a low education. These SES was expressed as a score (range -2.95 to 5.24 ) with a higher SES score representing a lower socioeconomic status $(26,28,29)$.

\section{Laboratory Measurements}

Ferritin was determined on an immunochemistry analyzer (Beckman Coulter, Fullerton, CA). STfR was determined by an ELISA technique (Ramco, Houston, TX). CRP was determined using a standard clinical chemistry analyzer (Beckman Coulter or F. Hoffmann-La Roche, Basel, Switzerland). Hb, MCV and Ret-Hb were determined using automated hematology analyzers (Sysmex Corporation, Kobe, Japan). We measured serum hepcidin by mass spectrometry (MS-hepcidin) and an immunochemical (IC) assay (Bachem, San Carlos) (IC-hepcidin) after one and two freeze-thaw cycles, respectively. The results of both methods were expressed in nanomoles per liter $(\mathrm{nmol} / \mathrm{l})(1 \mathrm{nmol} / \mathrm{l}=2.789 \mu \mathrm{g} / \mathrm{l})(2)$.

MS-hepcidin-25 measurements were performed by a combination of weak cation exchange chromatography and time-of-flight mass spectrometry (30). An internal standard (synthetic heavy hepcidin-25 stable isotope +40 ; custom made Peptide International) was used for quantification (22). Peptide spectra were generated on a Microflex LT matrix-enhanced laser desorption/ionisation TOF MS platform (Bruker Daltonics, Bremen, Germany). The lower detection limit of this method was $0.5 \mathrm{nmol} / \mathrm{l}$; average coefficients of variation were 2.8 $\%$ (intra-run) and $6.4 \%$ (inter-run) (22).

IC-Hepcidin-25 was determined by enzyme-linked immunoassay (ELISA) (Human hepcidin-25, extraction-free, EIA kit, Cat. No S-1337, Batch No A11599, Bachem, Peninsula Laboratories, San Carlos) in accordance with the manufacturer's instructions. According to the manufacturer this ELISA is specific for hepcidin-25. Before analysis the serum samples were diluted 1:6 in treated human serum provided with the kit. Hepcidin-25 levels were calculated from a calibration curve with a linear measuring range near the $\mathrm{IC}_{50}$ of the curve $(1.5 \mathrm{ng} / \mathrm{ml}$, range $0.35-1.72)$. Samples with CVs above $15 \%$ or with levels falling outside the measuring range were re-assayed in a different dilution. We calculated an intra- and inter-assay variation of $<10 \%$ and $<15 \%$, respectively.

\section{Statistical Analysis}

SPSS (version 18.0; SPSS, Chicago, IL) was used for statistical analysis. Before analysis data were checked on normality using histograms and Kolmogorov-Smirnov test. Hepcidin concentrations were right-skewed. For all continuous variables, median and 2.5th and 97.5th percentiles (P2.5 and P97.5 respectively) were calculated. For MS-hepcidin concentrations below the limit of detection $(0.5 \mathrm{nmol} / \mathrm{l})$ we used a value of 1.5 times the limit of detection $(0.25$ nmol/l). Blood sampling time and CRP were categorized in two groups based on clinically relevant cutoffs. For blood sampling time, these were before and after $12 \mathrm{pm}$ according to Dutch routine and in line with previously reported serum hepcidin concentration patterns throughout the day $(15,16,31)$. For CRP we used a cut-off value of less or more than $5 \mathrm{mg} / \mathrm{l}$ (32). Categorical variables were expressed in numbers and corresponding percentages. Reference ranges for MS-hepcidin and IC-hepcidin concentrations were constructed using the median, P2.5 and P97.5 in children of the iron-replete normative population. However, all children were analyzed to assess correlations of hepcidin with ferritin and CRP. Spearman correlation, intraclass correlation and Bland-Altman analysis were used to determine the relationship and differences between IC- and MS-hepcidin concentrations. Univariable and multivariable linear regression analysis were used to evaluate the associations with MS-hepcidin and IC-hepcidin as dependent variables and ferritin, $\mathrm{Hb}, \mathrm{MCV}$, Ret-Hb, and sTfR as independent variables, unadjusted and adjusted for age and time of blood sampling. The assumption of linearity between MS-hepcidin, IC-hepcidin concentrations and independent variables was confirmed using graphic methods. Resulting regression coefficients $(\beta)$ express the change in MS-hepcidin and IC-hepcidin concentration that were associated with a 1-unit change in the independent variable. The $R^{2}$ (adjusted for the number of explanatory variables in the model) was obtained to indicate the amount of variance in MS- and IC-hepcidin concentrations that were explained by the included variables. Statistical significance was defined as $P$ value $<0.05$.

\section{SUPPLEMENTARY MATERIAL}

Supplementary material is linked to the online version of the paper at http:// www.nature.com/pr

\section{STATEMENT OF FINANCIAL SUPPORT}

No external funding was used for this study. The authors did not receive any financial assistance to conduct this study or write this manuscript.

Disclosure: D.W.S. is "medical director" of the hepcidinanalysis.com initiative, through which the Radboudumc provides high quality hepcidin measurements for the scientific and medical community on a fee for service basis.

\section{REFERENCES}

1. Nemeth E, Ganz T. The role of hepcidin in iron metabolism. Acta Haematol 2009;122:78-86.

2. Kroot JJ, Tjalsma H, Fleming RE, Swinkels DW. Hepcidin in human iron disorders: diagnostic implications. Clin Chem 2011;57:1650-69.

3. Ganz T, Nemeth E. Regulation of iron acquisition and iron distribution in mammals. Biochim Biophys Acta 2006;1763:690-9.

4. Pak M, Lopez MA, Gabayan V, Ganz T, Rivera S. Suppression of hepcidin during anemia requires erythropoietic activity. Blood 2006;108:3730-5.

5. Ganz T, Nemeth E. Iron sequestration and anemia of inflammation. Semin Hematol 2009;46:387-93.

6. Weiss G, Goodnough LT. Anemia of chronic disease. N Engl J Med 2005;352:1011-23.

7. Müller KF, Lorenz L, Poets CF, Westerman M, Franz AR. Hepcidin concentrations in serum and urine correlate with iron homeostasis in preterm infants. J Pediatr 2012;160:949-53.e2.

8. Berglund S, Lönnerdal B, Westrup B, Domellöf M. Effects of iron supplementation on serum hepcidin and serum erythropoietin in low-birthweight infants. Am J Clin Nutr 2011;94:1553-61.

9. de Mast Q, Syafruddin D, Keijmel S, et al. Increased serum hepcidin and alterations in blood iron parameters associated with asymptomatic P. falciparum and P. vivax malaria. Haematologica 2010;95:1068-74.

10. Jaeggi T, Moretti D, Kvalsvig J, et al. Iron status and systemic inflammation, but not gut inflammation, strongly predict gender-specific concentrations of serum hepcidin in infants in rural Kenya. PLoS ONE 2013;8:e57513.

11. Semrin G, Fishman DS, Bousvaros A, et al. Impaired intestinal iron absorption in Crohn's disease correlates with disease activity and markers of inflammation. Inflamm Bowel Dis 2006;12:1101-6.

12. del Giudice EM, Santoro N, Amato A, et al. Hepcidin in obese children as a potential mediator of the association between obesity and iron deficiency. J Clin Endocrinol Metab 2009;94:5102-7.

13. Choi HS, Song SH, Lee JH, Kim HJ, Yang HR. Serum hepcidin levels and iron parameters in children with iron deficiency. Korean J Hematol 2012;47:286-92. 


\section{Articles | Uijterschout et al.}

14. Cangemi G, Pistorio A, Miano M, et al. Diagnostic potential of hepcidin testing in pediatrics. Eur J Haematol 2013;90:323-30.

15. Schaap CC, Hendriks JC, Kortman GA, et al. Diurnal rhythm rather than dietary iron mediates daily hepcidin variations. Clin Chem 2013;59:52735.

16. Kroot JJ, Kemna EH, Bansal SS, et al. Results of the first international round robin for the quantification of urinary and plasma hepcidin assays: need for standardization. Haematologica 2009;94:1748-52.

17. Berglund SK, Westrup B, Hägglöf B, Hernell O, Domellöf M. Effects of iron supplementation of LBW infants on cognition and behavior at 3 years. Pediatrics 2013;131:47-55.

18. Galesloot TE, Vermeulen SH, Geurts-Moespot AJ, et al. Serum hepcidin: reference ranges and biochemical correlates in the general population. Blood 2011;117:e218-25.

19. Kroot JJ, van Herwaarden AE, Tjalsma H, Jansen RT, Hendriks JC, Swinkels DW. Second round robin for plasma hepcidin methods: first steps toward harmonization. Am J Hematol 2012;87:977-83.

20. Hunter HN, Fulton DB, Ganz T, Vogel HJ. The solution structure of human hepcidin, a peptide hormone with antimicrobial activity that is involved in iron uptake and hereditary hemochromatosis. J Biol Chem 2002;277:37597-603.

21. Jordan JB, Poppe L, Haniu M, et al. Hepcidin revisited, disulfide connectivity, dynamics, and structure. J Biol Chem 2009;284:24155-67.

22. Laarakkers CM, Wiegerinck ET, Klaver S, et al. Improved mass spectrometry assay for plasma hepcidin: detection and characterization of a novel hepcidin isoform. PLoS ONE 2013;8:e75518.
23. Sherriff A, Emond A, Hawkins N, Golding J. Haemoglobin and ferritin concentrations in children aged 12 and 18 months. ALSPAC Children in Focus Study Team. Arch Dis Child 1999;80:153-7.

24. Looker AC, Dallman PR, Carroll MD, Gunter EW, Johnson CL. Prevalence of iron deficiency in the United States. JAMA 1997;277:973-6.

25. Uijterschout L, Vloemans J, Vos R, et al. Prevalence and risk factors of iron deficiency in healthy young children in the southwestern Netherlands. J Pediatr Gastroenterol Nutr 2014;58:195-200.

26. Mast AE, Blinder MA, Dietzen DJ. Reticulocyte hemoglobin content. Am J Hematol 2008;83:307-10.

27. Fredriks AM, van Buuren S, Burgmeijer RJ, et al. Continuing positive secular growth change in The Netherlands 1955-1997. Pediatr Res 2000;47:316-23.

28. Knol FA. From high to low; from low to high [in Dutch], 2012. (http:// www.scp.nl/Publicaties/Alle_publicaties/Publicaties_2012/Statusontwikkeling_van_wijken_in_Nederland_1998_2010.)

29. World Health Organization. Assessing the iron status of populations, 2007. (http://www.who.int/nutrition/publications/micronutrients/anaemia_ iron_deficiency/9789241596107/en/.)

30. Kroot JJ, Laarakkers CM, Geurts-Moespot AJ, et al. Immunochemical and mass-spectrometry-based serum hepcidin assays for iron metabolism disorders. Clin Chem 2010;56:1570-9.

31. Ganz T, Olbina G, Girelli D, Nemeth E, Westerman M. Immunoassay for human serum hepcidin. Blood 2008;112:4292-7.

32. Van den Bruel A, Thompson MJ, Haj-Hassan T, et al. Diagnostic value of laboratory tests in identifying serious infections in febrile children: systematic review. BMJ 2011;342:d3082. 